\title{
Role of Hedgehog-Glil signaling in the enhanced proliferation and differentiation of MG63 cells enabled by hierarchical micro-/nanotextured topography
}

\author{
This article was published in the following Dove Press journal: \\ International Journal of Nanomedicine \\ 20 April 2017 \\ Number of times this article has been viewed
}

Yao Lin'

Yinghe Huang'

Junbing $\mathrm{He}^{2}$

Feng Chen ${ }^{2}$

Yanfang $\mathrm{He}^{2}$

Wenying Zhang ${ }^{2}$

'Department of Stomatology, Taishan People's Hospital, Affiliated to Guangdong Medical University, Taishan, ${ }^{2}$ Guangdong Key Laboratory of Age-Related Cardiac and Cerebral Diseases, Institute of Neurology, Affiliated Hospital of Guangdong Medical University, Zhanjiang, Guangdong, People's Republic of China
Correspondence: Yinghe Huang Department of Stomatology,

Taishan People's Hospital, Affiliated to Guangdong Medical University, 80 Huanbei Avenue, Taicheng Town, Taishan, Guangdong 529200, People's Republic of China Tel +86 I 37022 I 8459 Fax +86750555 2059 Email yinghehuang_gmc@163.com
Abstract: Hedgehog-Gli1 signaling is evolutionarily conserved and plays an essential role in osteoblast proliferation and differentiation as well as bone formation. To evaluate the role of the Hedgehog-Glil pathway in the response of osteoblasts to hierarchical biomaterial topographies, human MG63 osteoblasts were seeded onto smooth, microstructured, and micro-/nanotextured topography (MNT) titanium to assess osteoblast proliferation and differentiation in terms of proliferative activity, alkaline phosphatase (ALP) production, and osteogenesis-related gene expression. Quantitative real-time polymerase chain reaction (qRT-PCR) was used to detect the mRNA expression of Sonic hedgehog (Shh), Smoothened (Smo), and Gli1, and the protein levels were assayed by Western blotting. MG63 cells treated with the Smo inhibitor cyclopamine were seeded onto the titanium specimens, and the cell proliferation and differentiation were studied in the presence or absence of cyclopamine. Our results showed that compared to the smooth and microstructured surfaces, the MNTs induced a higher gene expression and protein production of Shh, Smo, and Gli1 as well as the activation of Hedgehog signaling. The enhanced proliferative activity, ALP production, and expression of the osteogenesis-related genes (bone morphogenetic protein-2, ALP, and runt-related transcription factor 2) enabled by the MNTs were significantly downregulated by the presence of cyclopamine to a similar level as those on the smooth and acid-etched microstructured surfaces in the absence of cyclopamine. This evidence explicitly demonstrates pivotal roles of Hedgehog-Gli1 signaling pathway in mediating the enhanced effect of MNTs on MG63 proliferation and differentiation, which greatly advances our understanding of the mechanism involved in the biological responsiveness of biomaterial topographies. These findings may aid in the optimization of hierarchical biomaterial topographies targeting Hedgehog-Gli1 signaling.

Keywords: Hedgehog-Gli1, MG63, proliferation, differentiation, micro-/nanotextured topography

\section{Plain language summary}

The Hedgehog-Gli1 signaling plays pivotal roles in osteoblast proliferation, differentiation, and bone formation. However, little is known about the role of the Hedgehog signaling in osteoblast proliferation and differentiation by the micro-/nanotextured topographies (MNTs). In this study, we produced MNTs decorated with $\mathrm{TiO}_{2}$ nanotubes onto the titanium to evaluate the cell functions and Hedgehog-Gli1 signaling activation. We further blocked the Hedgehog-Gli1 pathway by using the Smoothened (Smo) inhibitor cyclopamine and monitored the downstream events including Gli1 protein levels and cell function. Our results showed that the MG63 cells seeded onto the MNTs exhibited significantly enhanced cell adhesion, proliferation, and 
differentiation compared to the other titanium surfaces. The treatment with cyclopamine decreased the proliferative activity of MG63 cells, especially the cells seeded onto the MNTs. Furthermore, the enhanced expressions of the osteogenesis-related genes and ALP production enabled by the MNTs were significantly downregulated by the presence of cyclopamine to a similar level as those on the smooth and acid-etched microstructured surfaces in the absence of cyclopamine. These results demonstrated pivotal roles of Hedgehog-Gli1 signaling pathway in mediating the enhanced proliferation and differentiation of MG63 osteoblasts by the MNTs, which might greatly advance our understanding on the mechanism implied in the biological responsiveness of biomaterial topographies.

\section{Introduction}

Natural bone tissues are hierarchical structures containing building blocks of various length scales, including nanoscale hydroxyapatite crystals, microscale fibrillar collagen, and macroscopic cancellous and cortical bones. ${ }^{1,2}$ Increasing evidence has demonstrated that the addition of hierarchical micro-/nanotextured topographies (MNTs) to a biomedical implant surface can effectively modulate various cellular functions, such as osteoblast attachment, proliferation, and differentiation, as MNTs can better imitate the hierarchical structures of natural bone tissues. ${ }^{3-5}$ However, the precise effect and mechanisms of the MNTs on the response of cells are still unclear, which has impeded the optimization of hierarchical biomaterial topographies.

The Hedgehog signaling pathway is evolutionarily conserved and plays an essential role in various developmental processes, such as osteoblast proliferation, differentiation, and maturation as well as bone formation. ${ }^{6-8}$ The Hedgehog family contains three protein ligands, including Sonic hedgehog (Shh), Indian hedgehog (Ihh), and Desert hedgehog (Dhh), which signal via a mechanism involving two transmembrane proteins, such as Smo and Patched homolog 1 (Ptch1). ${ }^{9}$ After a Hedgehog protein binds to Ptch1, Smo is rendered constitutively active and activates an intracellular signaling cascade, leading to the upregulated transcription of downstream genes such as Gli1. ${ }^{10,11}$ Recent studies indicated that the genomic deletion of Smo, which functions as a key transmembrane transducer in the Hedgehog signaling pathway, results in a significantly decreased osteogenic differentiation, a low expression of runt-related transcription factor 2 (Runx2), and primary spongiosa in mice. ${ }^{12,13}$ Consistent with this finding, Gli1 haploinsufficiency affects adult bone metabolism, which results in a decrease in bone mass and a delay in fracture healing in postnatal skeletons. ${ }^{14}$ Other studies demonstrated that the bone morphogenetic protein (BMP) signaling pathway acts downstream of Hedgehog signaling and modulates the differentiation of osteoblasts. ${ }^{15-17}$ The disruption of the Hedgehog pathway leads to various bone diseases, while the enhanced activity of the Hedgehog pathway in patients with bone fractures may promote bone repair and fracture healing. ${ }^{8}$

Recent studies have reported that the expression of several genes within the Hedgehog signaling pathway differed with different topographical surfaces of titanium. ${ }^{18,19}$ Several studies have further demonstrated that Hedgehog and Wnt signaling interact to modulate cartilage formation in a mouse model and osteoblast differentiation in modified titanium surfaces. ${ }^{19-22}$ The addition of MNTs to a biphasic calcium phosphate bone grafting material caused a significantly increased expression of Ihh and Gli1 and stronger bone formation compared to control tissue culture plastic. ${ }^{23}$ Other studies have shown that the presence of nanorods or nanoparticles on the substrates markedly upregulated the expression of a set of genes involved in the differentiation of osteoblast and the subsequent functions of cells, including Smo, Ihh, and Gli1. ${ }^{24,25}$ These data indicate a potential role of the Hedgehog signaling pathway in the enhanced osteoblast adhesion, growth, and differentiation via MNTs.

An increasing number of studies have demonstrated that several functional osteogenesis-related signaling pathways, such as the integrin-linked kinase/ $\beta$-catenin, extracellular signal-regulated kinase $1 / 2$, and Wnt, may contribute to the biological effects of micro-/nanotopographical cues in osteoblast differentiation and bone formation. ${ }^{26-28}$ However, to the best of our knowledge, little is known about the role of the Hedgehog signaling pathway in osteoblast proliferation and differentiation by MNTs. In this study, we produced hierarchical MNTs decorated with $\mathrm{TiO}_{2}$ nanotubes on the titanium through acid etching followed by anodization and evaluated the cell functions and Hedgehog-Gli1 signaling activation of human MG63 osteoblast cells on the MNTs. Moreover, we blocked the Hedgehog-Gli1 signaling pathway using the Smo inhibitor cyclopamine and, then, monitored the downstream events, including Gli1 protein levels and cell function.

\section{Methods \\ Specimen preparation}

This study was reviewed and approved by the Ethics Committee of the Affiliated Hospital of Guangdong Medical University, People's Republic of China. The pure titanium disks with a diameter of $21 \mathrm{~mm}$ and thickness of $1 \mathrm{~mm}$ to fit the 12-well tissue culture plates were cut from pure titanium plates. After polished using silicon carbide sandpapers of 280 , $360,400,600,800$, and 1,000 grits in series, the samples were treated with $1 \mathrm{wt} \%$ hydrofluoric (HF) acid for 15 minutes, then directly cleaned with double distilled $\mathrm{H}_{2} \mathrm{O}\left(\mathrm{ddH}_{2} \mathrm{O}\right)$ for 
10 minutes, and air dried at $37^{\circ} \mathrm{C}$. Next, they were anodized for 1 hour in an electrolyte containing ethylene glycol $(1,2-$ ethanediol, $95 \mathrm{vol} \%$ ) and $\mathrm{ddH}_{2} \mathrm{O}(5 \mathrm{vol} \%)+0.5 \mathrm{wt} \% \mathrm{NH}_{4} \mathrm{~F}$ using a direct-current regulated power supply with a platinum electrode as the cathode at two different voltages (10 and $20 \mathrm{~V}$ ) to form two typical hierarchical MNTs. The titanium samples were divided into the following four groups: polished smooth surface (S), acid-etched microstructured surface (R), acid-etched/anodized at $10 \mathrm{~V}$ (R10), and acid-etched/anodized at $20 \mathrm{~V}$ (R20). After being ultrasonically cleaned and air dried at room temperature, the titanium specimens were sterilized by cobalt-60 irradiation before being used in cell culture.

\section{Surface characterization of titanium}

The morphologies of the four different titanium surfaces were detected by field emission scanning electron microscopy (FESEM; S-4800; Hitachi Limited, Tokyo, Japan) according to previous studies. ${ }^{27,28}$ The measurement of titanium surface roughness was performed by the Micro-XAM-3D noncontact surface profiler (ADE Corporation, Westwood, MA, USA) in a noncontact mode. Two roughness parameters of the measurement at S, R, R10, and R20 titanium disks were as follows: average roughness $(\mathrm{Ra})$ and root mean square roughness $(\mathrm{Rq})$.

\section{Cell culture and reagents}

The human MG63 osteoblasts were obtained from ATCC (Rockville, MD, USA). The human MG63 osteoblasts were cultured in Dulbecco's Modified Eagle's Medium (DMEM; Thermo Fisher Scientific, Waltham, MA, USA) supplemented with $1 \%$ antibiotic mixture (penicillin/streptomycin; HyClone, Logan, UT, USA) and 10\% fetal bovine serum (FBS; Thermo Fisher Scientific) at $37^{\circ} \mathrm{C}$ under a $5 \% \mathrm{CO}_{2}$ humidified atmosphere. The MG63 osteoblasts were seeded onto the four different surfaces of titanium disks in 12-well plates with a concentration of $5 \times 10^{4} /$ well. The cyclopamine (MedChem Express, Princeton, NJ, USA) was dissolved at $5 \mathrm{mg} / \mathrm{mL}$ in dimethyl sulphoxide, and the solution was diluted to the final concentration with DMEM supplemented with $1 \%$ antibiotic mixture (penicillin/streptomycin) and 10\% FBS. Treatment with control vehicle was used for control cells.

\section{Cell adhesion assay}

The MG63 osteoblasts were cultured onto the four different titanium surfaces with a density of $1.3 \times 10^{4}$ cells $/ \mathrm{cm}^{2}$, and the initial adhesion of cells was assessed by counting the number of cells adhered to the titanium surfaces after 30 and 60 minutes of incubation. At each prescribed time point, the titanium substrates were rinsed gently with phosphate-buffered saline (PBS) solution to dislodge the nonadherent cells. Then, the $4 \%$ (w/v) paraformaldehyde was used to fix the cells attached to the titanium surfaces for 30 minutes. After rinsing gently with PBS, the nuclei of cells were stained with 2-(4-amidinophenyl)6-indolecarbamidine dihydrochloride (DAPI; Beyotime, Shanghai, People's Republic of China) for 5 minutes. The number of stained nuclei in ten random fields was counted under a fluorescence microscope to evaluate the cell adhesion $(400 \times$ magnification, counts performed over an area of $1,020 \times 800 \mu^{2}$; $n=3$, a total of 30 fields of view for each group).

\section{Cellular morphology onto different titanium surfaces}

After 8 hours of incubation, the titanium specimens were cleaned with PBS. The cells grown onto the S, R, R10, and R20 titanium surfaces were fixed in 4\% paraformaldehyde for 15 minutes and permeabilized with $0.1 \%$ Triton X-100 for 5 minutes at $37^{\circ} \mathrm{C}$ followed by two PBS rinses. Protected from light, the cellular actin cytoskeleton was then stained using the fluorescent dye rhodamine phalloidin (Thermo Fisher Scientific, Waltham, MA, USA) for 30 minutes, and the nuclei of cells were counterstained with DAPI for 1 minute. A fluorescence microscope was used to examine cellular morphology of MG63 osteoblasts seeded onto the different titanium surfaces.

\section{Cell proliferation assay}

The MG63 cells were seeded onto the different titanium surfaces in 12-well plates with a concentration of $5 \times 10^{4} /$ well and cultured for 1,3 , and 5 days to assess the cell proliferation by using the 3-(4,5-dimethylthiazol-2yl)-2,5-diphenyltetrazolium bromide (MTT; Sigma-Aldrich, St Louis, MO, USA) assay. The MG63 cells seeded onto the different titanium surfaces in 12 -well plates with a concentration of $5 \times 10^{4} /$ well were treated with $10 \mu \mathrm{M}$ of cyclopamine according to previous studies. ${ }^{29,30}$ The culture medium supplemented with cyclopamine was changed every 72 hours for a total period of 7 days, and then, the MTT assay was used to detect the proliferative activity of cells. At each prescribed time point, the titanium samples were gently cleaned twice with PBS and diverted to a new 12-well plate. The MTT solution was then added to each well, and the titanium samples were incubated at $37^{\circ} \mathrm{C}$ for 2 hours. The formazen was dissolved with dimethyl sulfoxide and measured on the spectrophotometer at $490 \mathrm{~nm}$.

\section{Alkaline phosphatase (ALP) staining and quantitative ALP assay}

The cyclopamine treatment processes were the same as those in the cell proliferation assay. The MG63 cells were seeded onto the different titanium surfaces in 12-well plates 
with a concentration of $5 \times 10^{4} /$ well for 7 days. Then, the cells were cleaned gently with PBS and fixed with $4 \%$ polyoxymethylene for 15 minutes. ALP staining was performed with a 5-bromo-4-chloro-3-indolyl phosphate (BCIP)/nitro blue tetrazolium (NBT) Alkaline Phosphatase Color Development Kit (Beyotime). The ALP activity was assessed using a quantitative ALP assay kit (Beyotime) according to the guidelines of the manufacturer. Briefly, the MG63 cells were cleaned twice with PBS and, then, lysed with ice-cold radioimmunoprecipitation (RIPA) lysis buffer (Beyotime). The aliquots of supernatants were added with substrates and $p$-nitrophenol and incubated for 15 minutes at $37^{\circ} \mathrm{C}$. The absorbance of $p$-nitrophenol formed was detected using a microplate reader at a wave length of $405 \mathrm{~nm}$. The amount of intracellular total protein was detected using the BCA Protein Assay Kit (Thermo Fisher Scientific) and the ALP activity was normalized to the total protein content reported as units per milligram of protein.

\section{RNA extraction and quantitative real-time polymerase chain reaction (qRT-PCR)}

The MG63 cells were cultured onto the different titanium surfaces in 12-well plates with a concentration of $5 \times 10^{4} /$ well and cultured for 3 and 7 days to detect the mRNA expression levels of Shh, Smo, Gli1, BMP-2, ALP, Runx2, and osteocalcin $(O C N)$. The cell culture and cyclopamine treatment processes were the same as those in the cell proliferation assay. The total RNA was isolated from the cultured cells with TRIzol (Sangon Biotech, Shanghai, People's Republic of China) and, then, was converted into cDNA by using the First Strand cDNA Synthesis Kit (Thermo Fisher Scientific) following the manufacturer's protocol. The mRNA expression levels of these genes were calculated by quantitative real-time PCR using the SYBR Green method. The primers designed using the Primer Premier 5.0 software (Premier Biosoft International, Palo Alto, CA, USA) and produced by Shanghai Sangon Company (Shanghai, People's Republic of China) are listed in Table 1. The PCR amplification was conducted in a $10 \mu \mathrm{L}$ final volume containing SYBRII Green PCR master mix ( $5 \mu \mathrm{L}$; TaKaRa Bio Inc, Kusatsu, Japan), each of specific forward and reverse primers $(0.2 \mu \mathrm{L}), \mathrm{ddH}_{2} \mathrm{O}$ $(3.6 \mu \mathrm{L})$, and cDNA $(1 \mu \mathrm{L})$. The quantitative real-time PCR was performed using a LightCycler 480 sequence detector system (Roche Applied Science, Laval, QC, Canada) in the following reaction conditions: $95^{\circ} \mathrm{C} / 300$ seconds, and 40 cycles of $95^{\circ} \mathrm{C} / 5$ seconds, $58^{\circ} \mathrm{C} / 20$ seconds, and $72^{\circ} \mathrm{C} / 60$ seconds. The relative expression levels for each gene of interest were normalized to that of glyceraldehyde3-phosphate dehydrogenase (GAPDH), calculated by the $2^{-\Delta \Delta \mathrm{Ct}}$ method.

\section{Western blot analysis}

The MG63 cells were seeded onto the four different titanium surfaces in 12 -well plates with a concentration of $5 \times 10^{4} /$ well, and cyclopamine treatment processes were the same as those in the cell proliferation assay. After 7 days of incubation, the total proteins were extracted from the cells using the RIPA lysis buffer. The concentrations of protein were detected using the BCA Protein Assay Kit. Then, the total proteins ( $25 \mu \mathrm{g}$ per lane) were separated by $10 \%$ sodium dodecyl sulfate-polyacrylamide gel electrophoresis and, then, transferred to polyvinylidene difluoride (PVDF) membranes (Millipore, Bedford, MA, USA). After blocking with $5 \%$ nonfat milk at $37^{\circ} \mathrm{C}$ for 2 hours, the membranes were incubated overnight at $4{ }^{\circ} \mathrm{C}$ with anti-Shh (rabbit monoclonal antibody, 1:500 diluted, ab53281; Abcam, Cambridge, UK), anti-Smo (rabbit polyclonal antibody, 1:800 diluted, sc-13943; Santa Cruz Biotechnology, Santa Cruz, CA, USA), anti-Gli1 (rabbit polyclonal antibody, 1:500 diluted, \#2534; CST, Danvers, MA, USA), and anti- $\beta$-actin antibody (1:2,000 diluted; Santa Cruz Biotechnology), followed by horseradish peroxidase-linked secondary antibodies. The immunoreactive bands were visualized by using an enhanced chemiluminescence (ECL) detection kit (Millipore).

Table I Primer sequences used for quantitative real-time PCR analysis of gene expression

\begin{tabular}{|c|c|c|}
\hline Gene & Forward primer sequence $\left(5^{\prime}-3^{\prime}\right)$ & Reverse primer sequence $\left(5^{\prime}-3^{\prime}\right)$ \\
\hline Shh & TGAAAGCAGGCAAGGAAAGGA & ACAAACTCTTGGCTCCGTCAA \\
\hline Smo & ACCTATGCCTGGCACACTTC & TTGCCACAGTGAGGACAAAG \\
\hline Gli I & GGCTGCACCAAACGCTATAC & TTTGGTCACATGGGCGTCAG \\
\hline BMP-2 & GGACGCTCTTTCAATGGACG & GCAGCAACGCTAGAAGACAG \\
\hline$A L P$ & CCTTGTAGCCAGGCCCATTG & GGACCATTCCCACGTCTTCAC \\
\hline OCN & TCCTTTGGGGTTTGGCCTAC & CCAGCCTCCAGCACTGTTTA \\
\hline Runx2 & CACTGGCGCTGCAACAAGA & CATTCCGGAGCTCAGCAGAATAA \\
\hline
\end{tabular}

Abbreviations: ALP, alkaline phosphatase; BMP-2, bone morphogenetic protein-2; OCN, osteocalcin; PCR, polymerase chain reaction; Runx2, runt-related transcription factor 2; Shh, Sonic hedgehog; Smo, Smoothened. 
The quantification of protein was calculated by densitometry analysis using the Quantity One 4.6.6 software.

\section{Statistical analyses}

Statistical analysis was performed using GraphPad Prism 4.0 (Graphpad Software Inc., San Diego, CA, USA) and SPSS Version 19.0 (IBM Corp., Armonk, NY, USA). All data were shown as the mean \pm standard error of the mean (SEM) from at least three independent experiments and analyzed by one-way analysis of variance combined with Student-Newman-Keuls post hoc test, or Student's $t$-test. The criterion for significance was set at $P<0.05$ for all the tests.

\section{Results}

\section{Characteristics of the titanium surface}

As shown in the SEM images (Figure 1), the multilevel pores were initially shaped onto the titanium surface; $10-40 \mu \mathrm{m}$ pits and $1-5 \mu \mathrm{m}$ micropits were created by etching in $1 \%$ $\mathrm{HF}$ acid for 15 minutes. After subsequent anodization at two different voltages ( 10 and $20 \mathrm{~V})$, two different diameters of $\mathrm{TiO}_{2}$ nanotubes ( 25 and $70 \mathrm{~nm}$ ) were shaped onto the microstructured surface and uniformly distributed. The surfaces of the smooth titanium disks were relatively smooth and planar compared to the other groups, consistent with previous reports. The surface roughness of the titanium disks was detected using a Micro-XAM-3D noncontact surface profiler on large scanning areas of $\sim 1.8 \times 1.4 \mathrm{~mm}$ and $450 \times 350 \mu \mathrm{m}$
(Figure 2). The profile topography measurements exhibited significant differences in the roughness parameters $\mathrm{Ra}$ and $\mathrm{Rq}$ between the smooth titanium group and the other titanium groups $(P<0.001)$. However, no significant differences in the surface roughness were observed among the R, R10, and R20 titanium groups.

\section{Cell adhesion}

Early cell adhesion onto the different titanium substrates during the first 30 and 60 minutes of culturing is presented in Figure 3. At each prescribed time point, the number of cells attached to the microstructured (R) and $\mathrm{TiO}_{2}$ micro-/nanotextured titanium surfaces (R10 and R20) was significantly larger than that on the smooth titanium surface $(P<0.05)$. No significant differences in the number of adherent cells were observed between the acid-etched microstructured and the $\mathrm{TiO}_{2}$ micro-/ nanotextured titanium surfaces $(P>0.05)$.

\section{Cellular morphology on the different titanium surfaces}

The cellular morphology of the different titanium surfaces after the first 8 hours of incubation is presented in Figure 4. The fluorescence microscopic images showed that the cells with spindle shapes spread on the smooth titanium surface, while the cells cultured on the other titanium surfaces (R, R10, and R20) displayed a larger and flattened morphology. The cells on the two $\mathrm{TiO}_{2}$ micro-/nanotextured titanium surfaces (R10
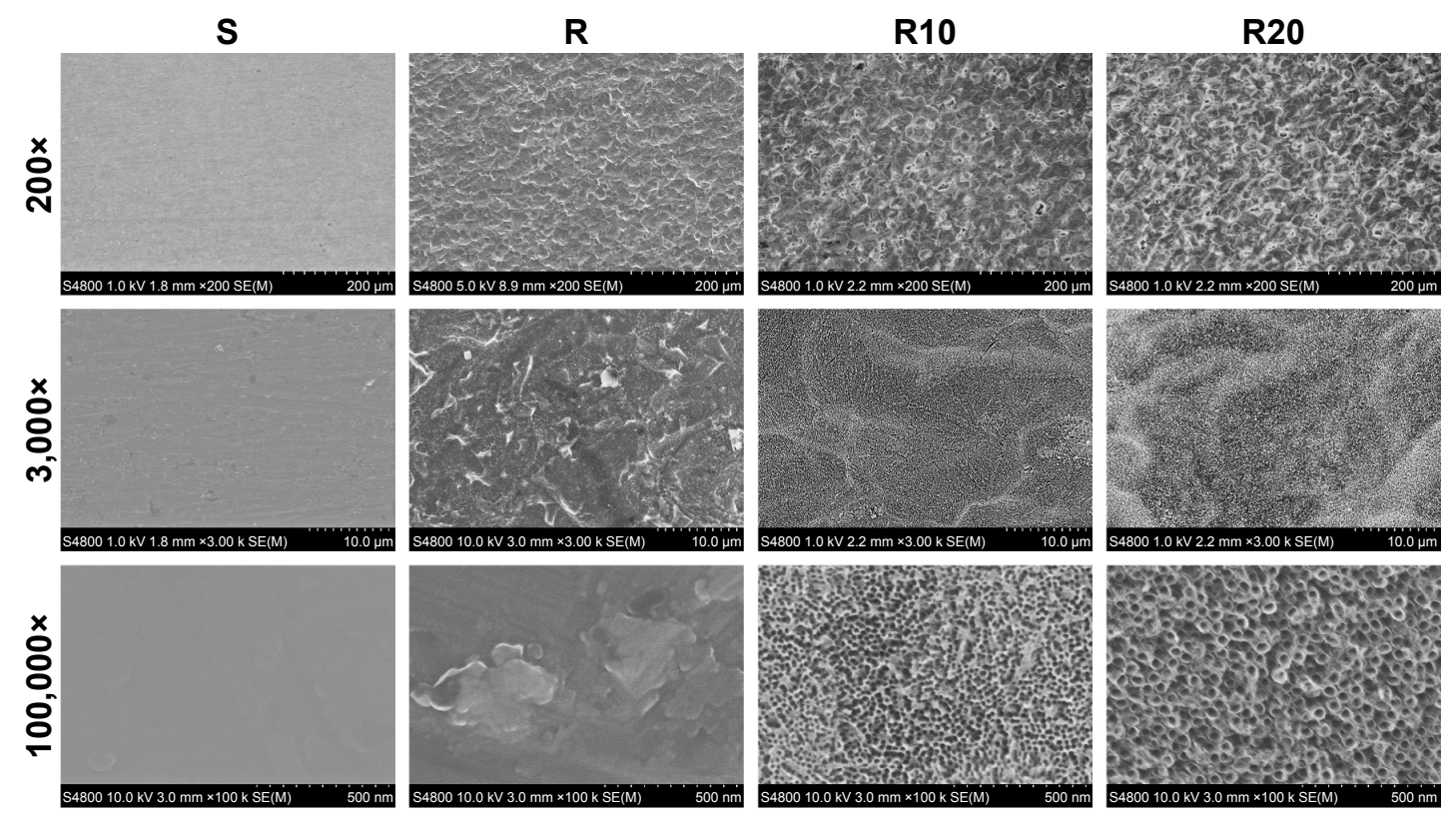

Figure I Representative FE-SEM pictures of the smooth (S), acid-etched microstructured (R), and hierarchical micro-/nanotextured surfaces (RI0 and R20). Notes: The nanotubes were found to have 25 and $70 \mathrm{~nm}$ outer diameters. Nanotubes of different sizes were formed on the microstructured surface and were distributed relatively uniformly.

Abbreviation: FE-SEM, field emission scanning electron microscopy. 


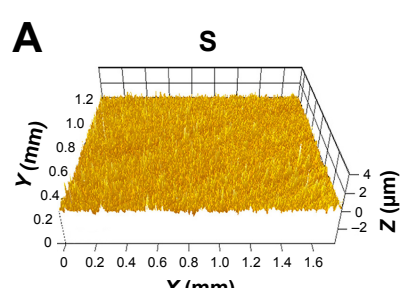

$X(\mathrm{~mm})$

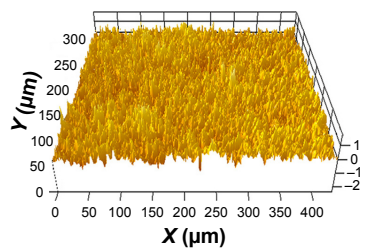

$\mathbf{R}$

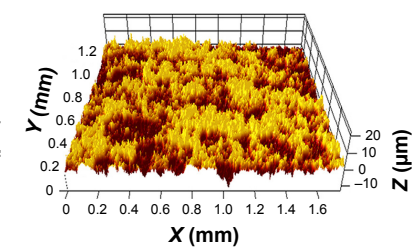

$X(\mathrm{~mm})$

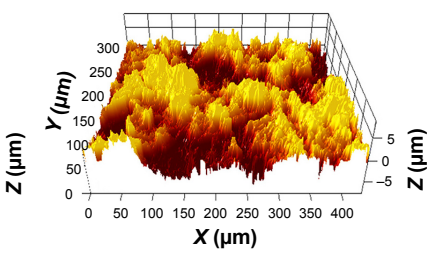

$\boldsymbol{X}(\mu \mathrm{m})$

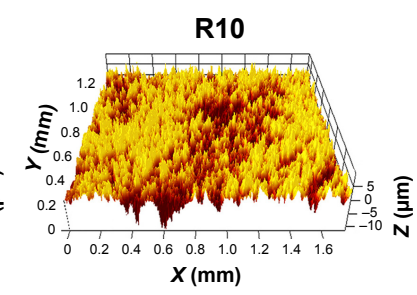

$X(\mathrm{~mm})$

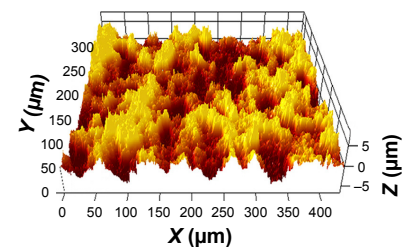

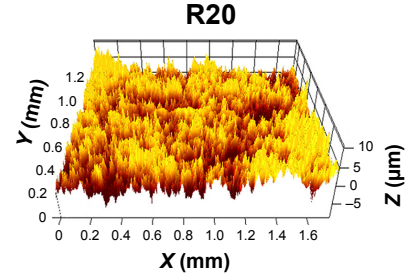

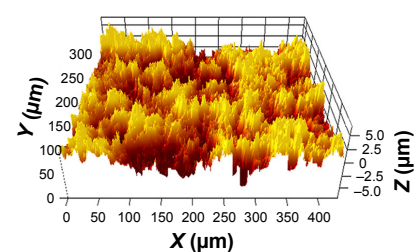

B

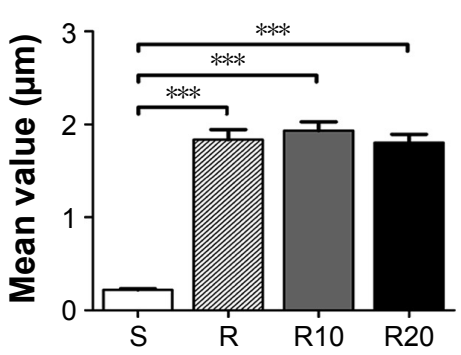

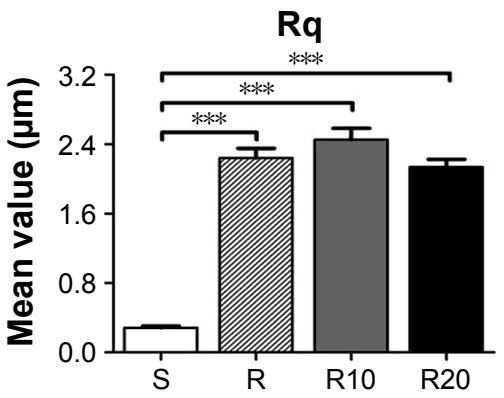

Figure 2 Micro-XAM-3D noncontact profiler surface roughness analysis of the titanium disks.

Notes: (A) Representative Micro-XAM-3D noncontact profiler pictures of the smooth (S), acid-etched microstructured (R), and hierarchical micro-/nanotextured surfaces (RI0 and R20) on large scanning areas of $1.8 \mathrm{~mm} \times 1.4 \mathrm{~mm}$ and $450 \mu \mathrm{m} \times 350 \mu \mathrm{m}$, respectively. (B) Comparisons of the roughness parameter (Ra and Rq) of different titanium surfaces. $* * * P<0.001$.

Abbreviations: Ra, average roughness; Rq, root mean square roughness.
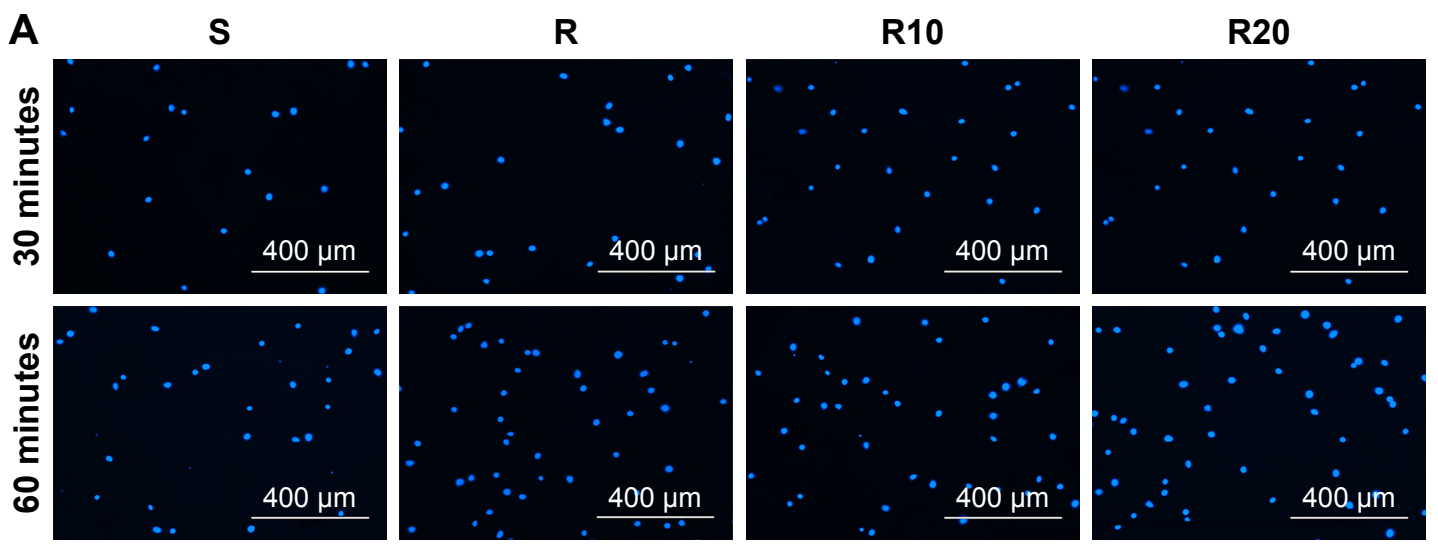

B

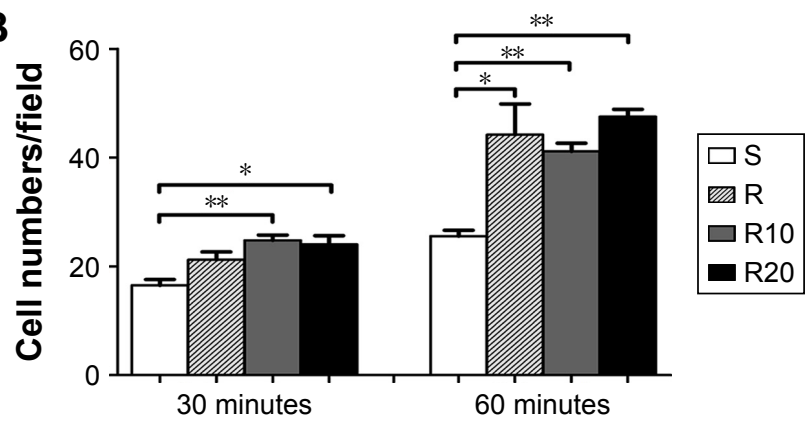

Figure 3 The initial adhesion of MG63 osteoblasts evaluated by counting the number of cell nuclei stained by DAPI under a fluorescence microscope after 30 and 60 minutes of incubation.

Notes: (A) The fluorescence microscope images of stained cells. (B) Mean \pm SEM cell numbers were calculated (400× magnification, counts performed over an area of $1,020 \times 800 \mu \mathrm{m}^{2} ; n=3$, a total of 30 fields of view for each group of the smooth [S], acid-etched microstructured [R], and hierarchical micro-/nanotextured surfaces [RI0 and R20]). $* P<0.05 ; * * P<0.01$.

Abbreviations: DAPI, 2-(4-amidinophenyl)-6-indolecarbamidine dihydrochloride; SEM, standard error of the mean. 

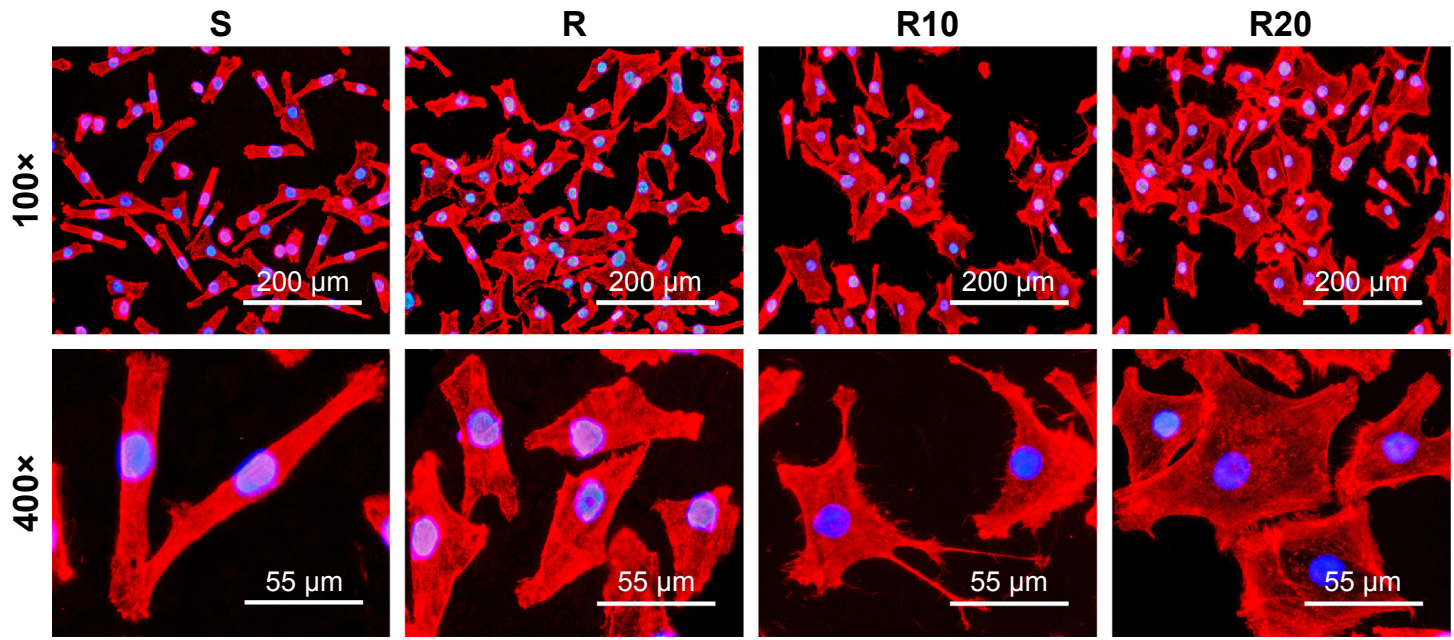

Figure 4 Representative fluorescence microscope images of cells stained with rhodamine phalloidin for actin filaments (red) and DAPI for cell nucleus (blue) after 8 hours of incubation onto the smooth (S), acid-etched microstructured (R), and hierarchical micro-/nanotextured surfaces (RI0 and R20).

Notes: The cells with spindle shape spread on the smooth surface, while the cells on the other surfaces showed a rather larger and flattened morphology. The cells on the $\mathrm{TiO}_{2}$ micro-/nanotextured titanium surfaces (RIO and R20) extended numerous pseudopodia of filament or sheet shape and spread properly on these surfaces.

Abbreviation: DAPI, 2-(4-amidinophenyl)-6-indolecarbamidine dihydrochloride.

and R20) extended numerous filamentous pseudopodia or had a sheet shape and spread properly on these surfaces.

\section{MG63 proliferation and differentiation on the different titanium surfaces}

Proliferation and differentiation were monitored to evaluate the response of MG63 cells to the different titanium surfaces. Cell proliferation was evaluated at days 1,3 , and 5 using an
MTT assay (Figure 5A). Cell proliferation increased over time in all four groups. At day 1, there was no significant difference among the four groups. However, at both days 3 and 5, the $\mathrm{TiO}_{2}$ micro-/nanotextured surfaces (R10 and R20) induced significantly higher proliferative activity than the other groups. A quantitative ALP assay and ALP staining performed after 7 days of incubation demonstrated that the MNTs (R10 and R20) induced a significantly higher ALP production than the
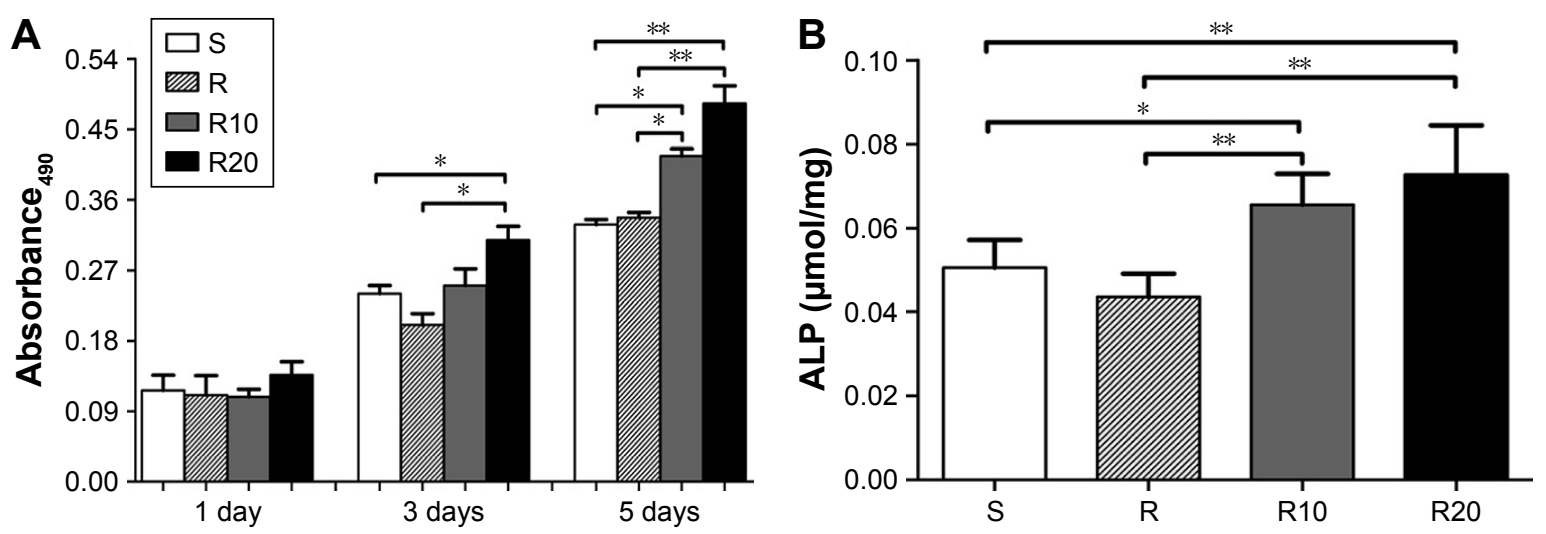

C

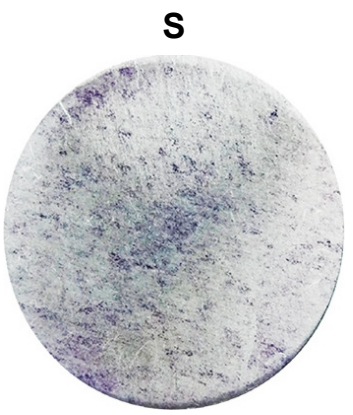

$\mathbf{R}$

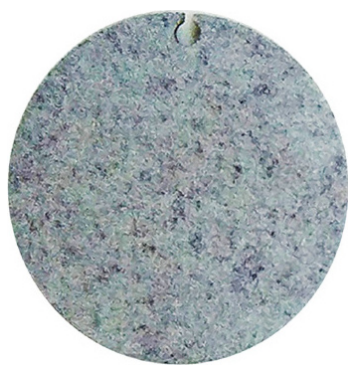

R10

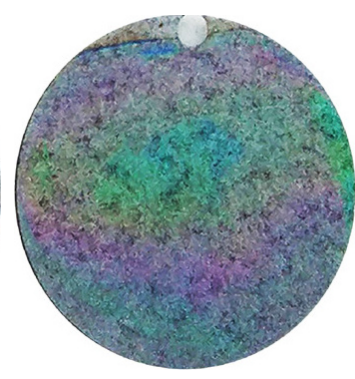

R20

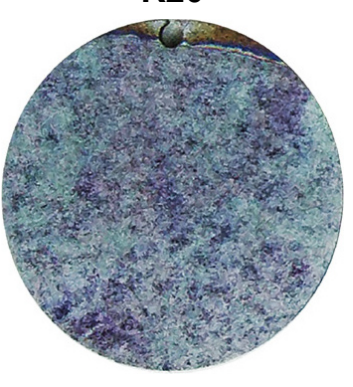

Figure 5 (Continued) 
D

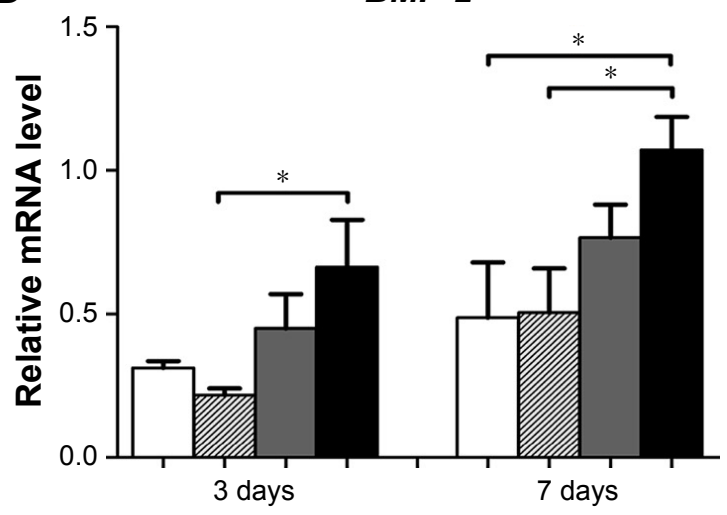

$\mathbf{F}$

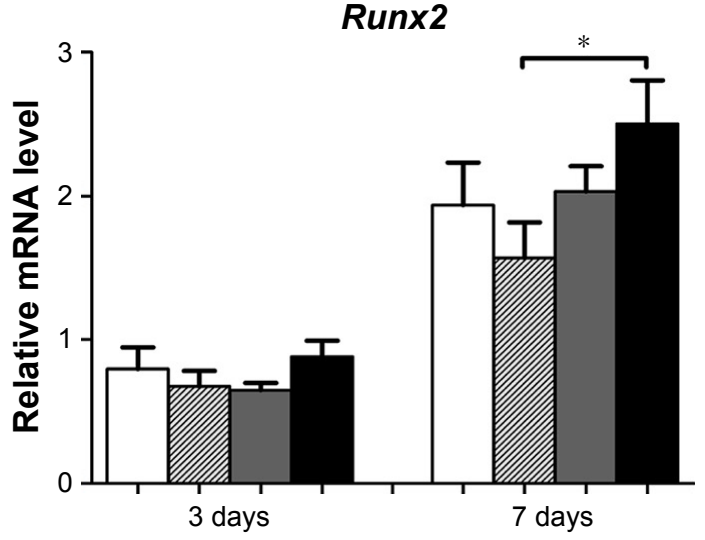

E

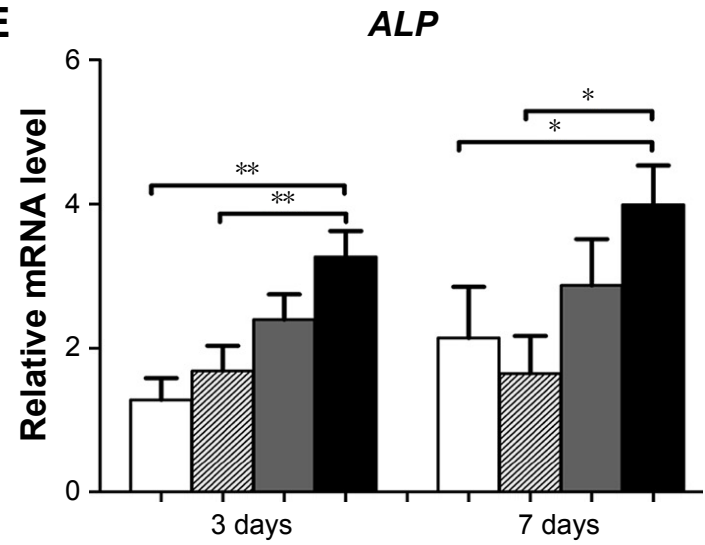

G

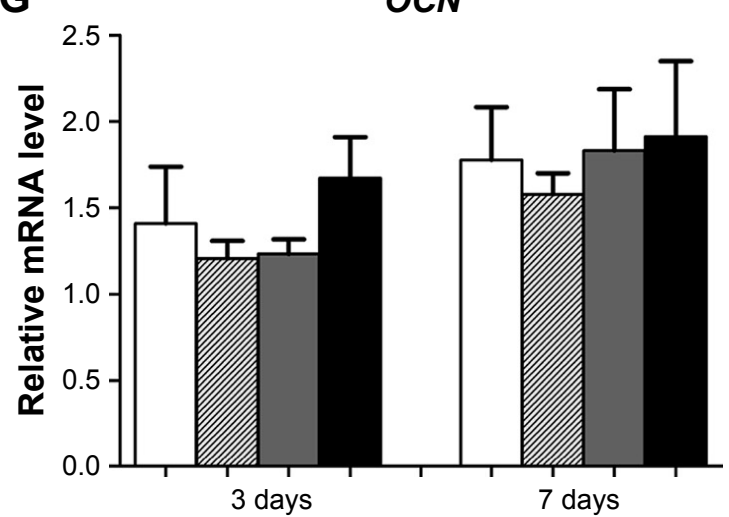

$\square S \quad \square R 10 \quad \square R 20$

Figure 5 The proliferation and differentiation of MG63 osteoblasts on the smooth (S), acid-etched microstructured (R), and hierarchical micro-/nanotextured surfaces (RI0 and R20).

Notes: (A) The proliferation of MG63 cells seeded onto the titanium surfaces was measured by the MTT assay. (B) A quantitative ALP activity standardized relative to the intracellular total protein content after 7 days of incubation is presented. (C) ALP activity evaluated by the ALP staining after 7 days of incubation is also presented. The expression of BMP-2 (D), ALP (E), Runx2 (F), and OCN (G) in MG63 osteoblasts after 3 and 7 days of incubation was detected by quantitative real-time PCR. $* P<0.05$; $* * P<0.01$.

Abbreviations: ALP, alkaline phosphatase; BMP-2, bone morphogenetic protein-2; MTT, 3-(4,5-dimethylthiazol-2yl)-2,5-diphenyltetrazolium bromide; OCN, osteocalcin; PCR, polymerase chain reaction; Runx2, runt-related transcription factor 2.

smooth and acid-etched microstructured surfaces, and R20 induced the highest levels (Figure 5B and C). Quantitative real-time PCR was used to evaluate the mRNA expression levels of several osteogenesis-related genes, such as $B M P-2$, $A L P$, Runx2, and $O C N$. These data indicated that the $\mathrm{TiO}_{2}$ micro-/nanotextured titanium surfaces (R10 and R20) significantly upregulated the mRNA expression levels of $B M P-2$ and $A L P$ compared to the $\mathrm{S}$ and $\mathrm{R}$ titanium surfaces at days 3 and 7 , and R20 induced the highest levels (Figure 5D and E). For Runx2, upregulation by R20 was only observed at the 7-day time point (Figure 5F). No significant difference in the expression level of $O C N$ was observed among the four different titanium surfaces (Figure 5G). Taken together, these findings show that the $\mathrm{TiO}_{2}$ micro-/nanotextured titanium surfaces effectively promoted the proliferation and differentiation of MG63 cells, and R20 was the most effective.

\section{The activity of the Hedgehog-Glil} signaling pathway

The gene expression levels in the Hedgehog-Gli1 signaling pathway on the titanium surfaces were quantified using the quantitative real-time PCR (Figure 6). In general, the mRNA expression levels of Shh (Figure 6A), Smo (Figure 6B), and Gli1 (Figure 6C) in MG63 cells on the $\mathrm{S}$ titanium surface were similar to those on the R titanium surface after 3 and 7 days of culturing. However, cells seeded onto the $\mathrm{TiO}_{2}$ micro-/ nanotextured titanium surfaces (R10 and R20) exhibited significantly increased mRNA expression levels of Shh, Smo, and Glil at the two time points, and R20 induced the highest levels. The levels of related proteins in the Hedgehog-Gli1 signaling pathway were detected by Western blotting after 7 days of culturing. The R surface displayed the lowest level of Shh protein, whereas the R20 surface induced the highest, 

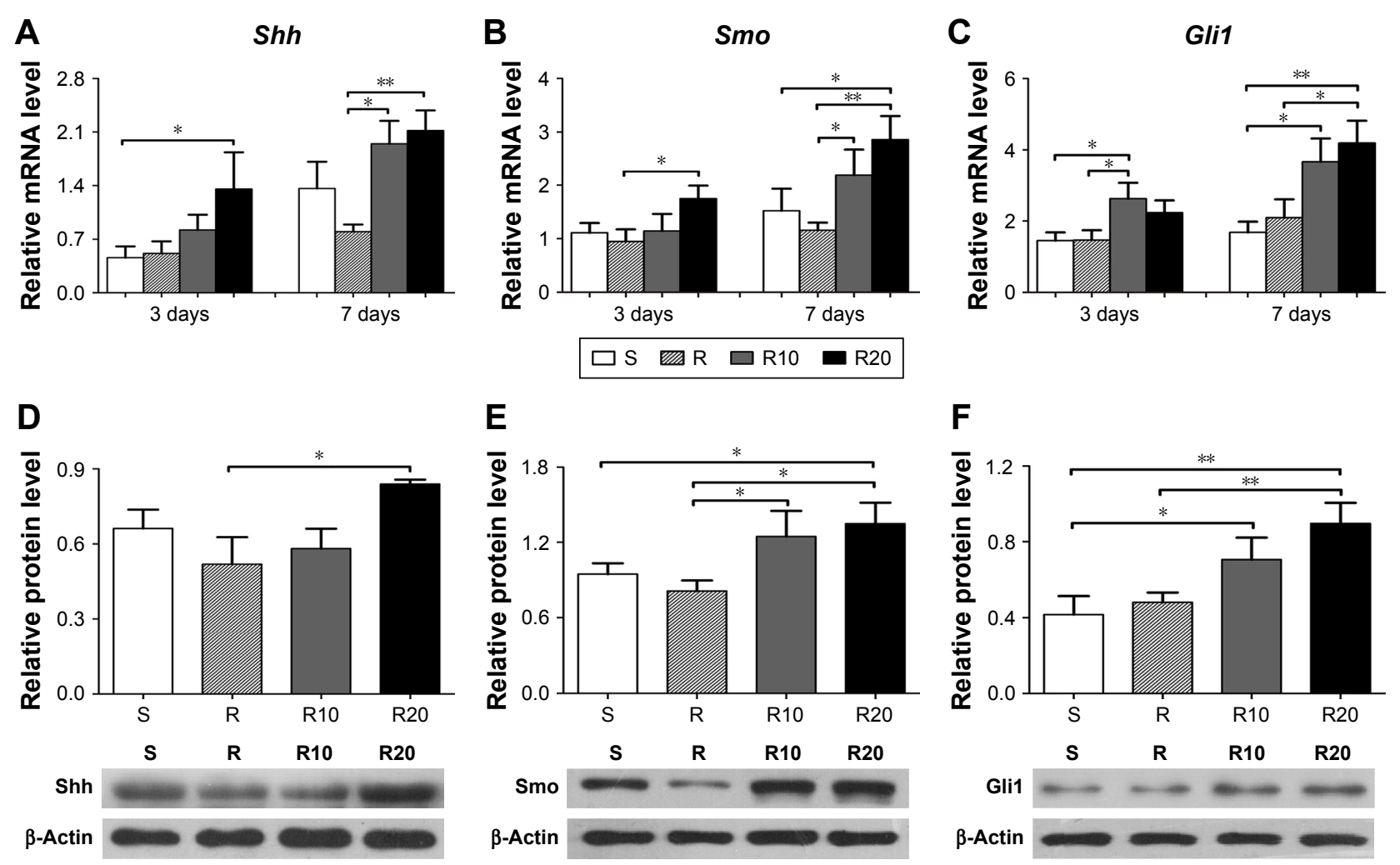

Figure 6 The activity of Hedgehog-Glil signaling pathway on the smooth (S), acid-etched microstructured (R), and hierarchical micro-/nanotextured surfaces (RI0 and R20). Notes: Quantitative real-time PCR analysis of Shh (A), Smo (B), and Glil (C) gene expressions in MG63 osteoblasts after 3 and 7 days of incubation; Western blot analysis of Shh (D), Smo (E), and Glil (F) protein levels in MG63 osteoblasts after 7 days of incubation. $* P<0.05 ; * * P<0.0$ I.

Abbreviations: PCR, polymerase chain reaction; Shh, Sonic hedgehog; Smo, Smoothened.

followed by the S surface (Figure 6D). Furthermore, the product of Smo, a key protein in the transmission of Hedgehog signaling across the plasma membrane, was significantly enhanced by the $\mathrm{TiO}_{2}$ micro-/nanotextured titanium surfaces, especially R20 (Figure 6E). Similarly, the R20 surface induced the highest level of Gli1 protein, followed by R10, whereas the R surface displayed a similar level of Glil to that of the smooth surface (Figure 6F). In general, the activity of the Hedgehog-Gli1 signaling pathway was sensitive to surface properties. The $\mathrm{TiO}_{2}$ micro-/nanotextured titanium surfaces activated the Hedgehog-Gli1 signaling pathway by stimulating the gene expression and protein production of Shh, Smo, and Gli1, and R20 had the greatest effect.

\section{Role of Hedgehog-Gli I signaling in MG63 proliferation and differentiation}

We further suppressed Hedgehog-Glil signaling using the Smo inhibitor cyclopamine to determine its role in the promoting effects of MNTs on MG63 proliferation and differentiation. As presented in Figure 7, our results showed that cyclopamine significantly suppressed the mRNA and protein levels of Smo as well as Gli1 nuclear accumulation after
7 days of incubation. Treatment with cyclopamine decreased the proliferative activity of MG63 cells, especially the cells seeded onto MNTs (Figure 8A). Treatment with cyclopamine markedly reduced the ALP production of cells seeded onto MNTs but slightly decreased the ALP production of cells seeded onto the $\mathrm{S}$ and R surfaces (Figure 8B). Furthermore, significantly decreased expression levels of $B M P-2$ and $A L P$ were observed in MG63 cells seeded onto the MNTs after treatment with cyclopamine, while the $B M P-2$ and $A L P$ expressions in cells on the $\mathrm{S}$ and $\mathrm{R}$ surfaces were not significantly affected by cyclopamine (Figure 8C and D). For the gene Runx2, significantly decreased expression levels were only observed on the R20 titanium surface but not on the R10 titanium surface (Figure 8E). Treatment with cyclopamine dramatically decreased the expression of BMP-2, ALP, and Runx 2 on MNTs to a similar level as those on the smooth and acid-etched microstructured surfaces in the absence of cyclopamine. However, no significant differences in the $O C N$ expression levels were observed between the cyclopamine-treated and untreated cells seeded onto the four different titanium surfaces (Figure 5F). In summary, Hedgehog-Gli1 signaling mediates the enhanced 

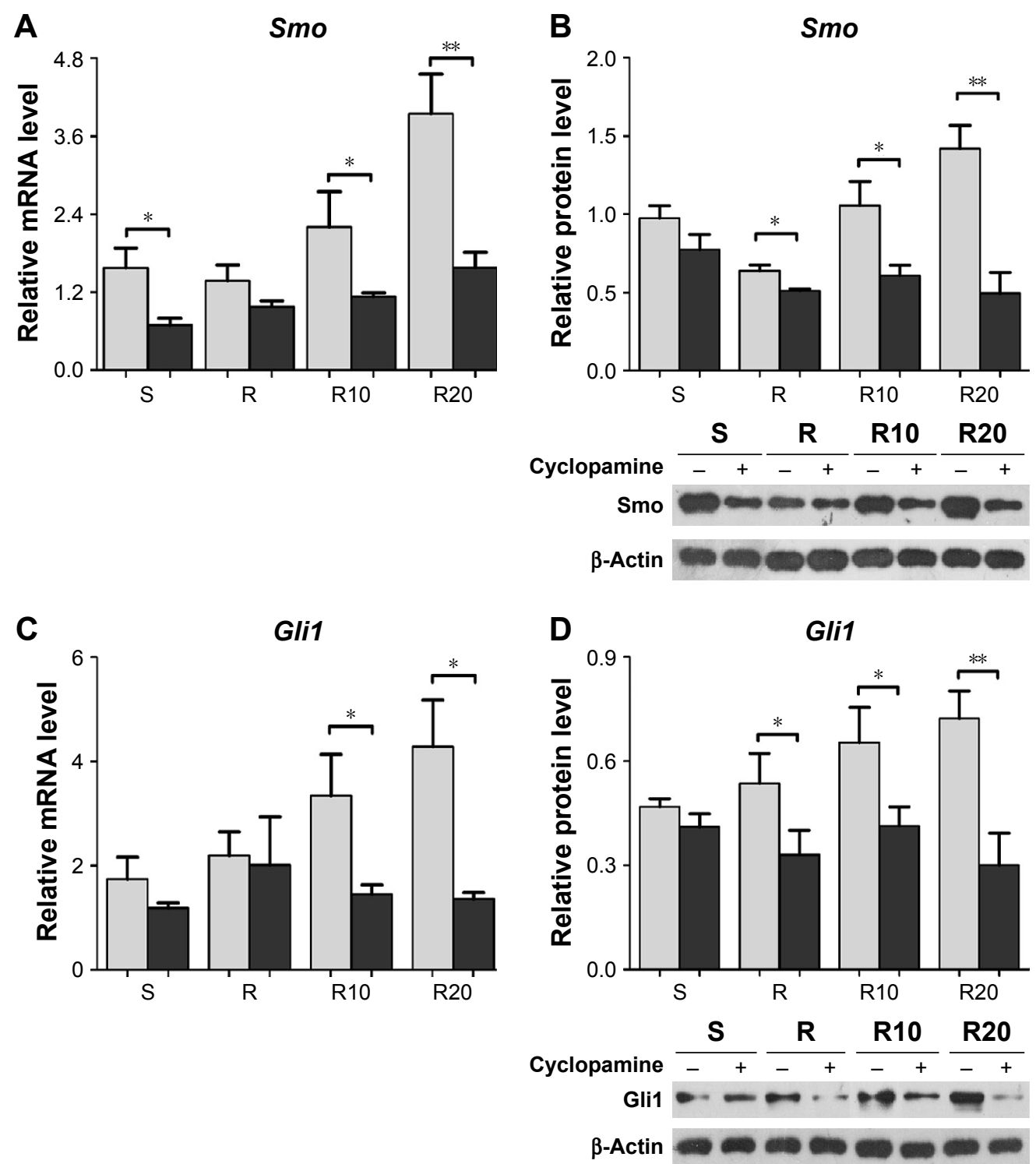

\section{$\square$ Control $\square$ Cyclopamine}

Figure 7 The Smo inhibitor cyclopamine suppressed the activity of Hedgehog-Glil signaling.

Notes: (A and B) The gene expression and protein production of Smo in MG63 osteoblasts after 7 days of incubation on the different titanium surfaces. (C and D) The gene expression and protein production of Glil in MG63 osteoblasts after 7 days of incubation on the different titanium surfaces. $* P<0.05 ; * * P<0.01$.

Abbreviation: Smo, Smoothened.

differentiation and differentiation of MG63 osteoblasts enabled by MNTs.

\section{Discussion}

Accumulating evidence has demonstrated that hierarchical MNTs on the surface of biomedical implants contribute to various cellular functions, such as osteoblast attachment, proliferation, and differentiation, as they can better imitate the hierarchical structures of natural bone tissues. ${ }^{3-5}$ However, the association between the hierarchical MNTs and their effects on regulating the functions of cells requires further exploration. Recent studies have shown that the activity of the Hedgehog signaling was sensitive to the surface properties of biomedical implants; the surface micro-/nanotexture on biomaterials induced a significant upregulation of Hedgehog-Gli1 signaling. ${ }^{18-20,23-25}$ Given the evidence implicating significant roles of the Hedgehog signaling in osteoblast proliferation and differentiation and bone formation, we investigated the role of Hedgehog signaling in the MG63 osteoblast proliferation and differentiation caused by MNTs. Our results indicated that the enhanced proliferation and differentiation of MG63 osteoblasts by MNTs are associated 


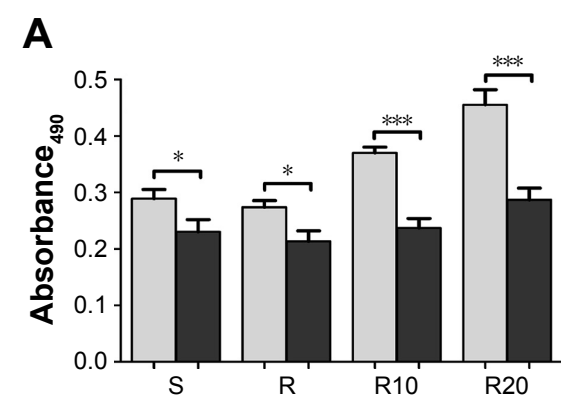

D

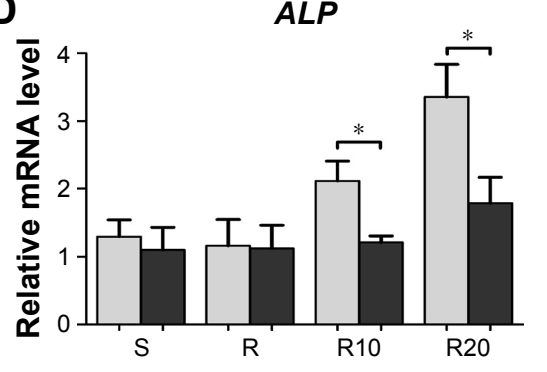

B

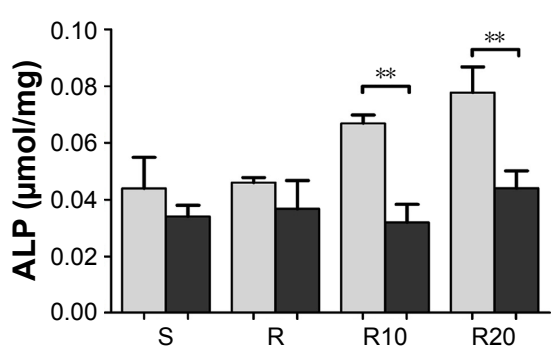

E

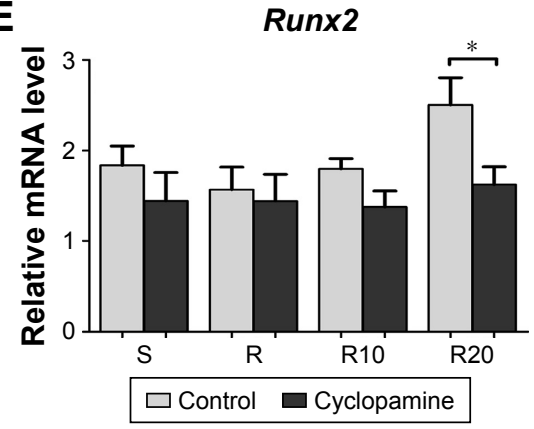

C

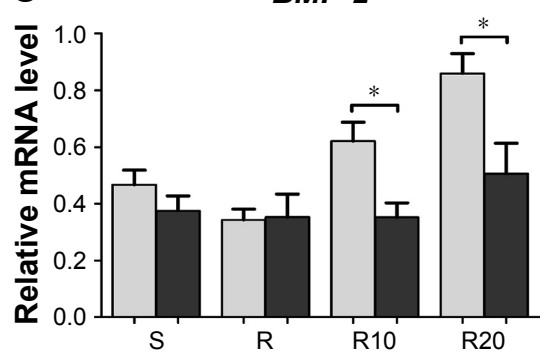

$\mathrm{F} \quad \mathrm{OCN}$

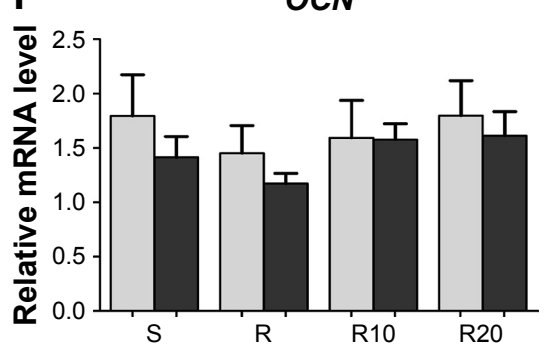

Figure 8 The effect of cyclopamine on the proliferation and differentiation of MG63 osteoblasts on the smooth (S), acid-etched microstructured (R), and hierarchical micro-/ nanotextured surfaces (RI0 and R20).

Notes: (A) The proliferation of cyclopamine-treated and untreated cells seeded onto the titanium surfaces as measured by the MTT assay after 7 days of incubation. (B) A quantitative ALP activity standardized relative to the intracellular total protein content after 7 days of incubation is presented. Quantitative real-time PCR analysis of BMP-2 $(\mathbf{C}), A L P(\mathbf{D})$, Runx2 (E), and $O C N(\mathbf{F})$ expressions in the cyclopamine-treated and untreated cells after 7 days of incubation on the different titanium surfaces. $* P<0.05$; $* * P<0.01 ; * * * P<0.001$.

Abbreviations: ALP, alkaline phosphatase; BMP-2, bone morphogenetic protein-2; MTT, 3-(4,5-dimethylthiazol-2yl)-2,5-diphenyltetrazolium bromide; OCN, osteocalcin; $\mathrm{PCR}$, polymerase chain reaction; Runx2, runt-related transcription factor 2.

with the increased gene expression and protein production of Shh, Smo, and Glil as well as the activation of Hedgehog signaling, suggesting that the biological effects of MNTs may be mediated by the Hedgehog signaling pathway.

Though biomaterial surfaces with rougher microtopography contribute to an enhanced early cell adhesion, osteoblast differentiation, and bone formation compared to a smooth surface, ${ }^{31,32}$ the early osseointegration of the implants and cell behaviors, including extracellular matrix deposition and mineralization, proliferation, and intracellular total protein synthesis, is relatively depressed on the microscale surface topographies. ${ }^{33-35}$ Several lines of evidence have demonstrated that the addition of nanotextured topography to the microstructured surface can effectively maintain and enhance the osteoblast attachment, proliferation, and differentiation and extracellular matrix deposition, as it can better imitate the hierarchical structure of bone tissues. ${ }^{3-5,36,37}$ Consistent with these previous studies, our results showed that the MG63 cells seeded onto MNTs exhibited a significantly enhanced cell adhesion, proliferation, and differentiation compared to smooth and microstructured surfaces. Immunofluorescent images of MG63 cells on the MNTs showed the formation of numerous filopodia, lamellipodia, and cellular extensions compared to those of cells grown on the smooth control surface. The R20 surface induced the highest proliferative activity, ALP production, and expression levels of osteogenesis-related genes, such as BMP-2, ALP and Runx2, followed by the R10 surface. However, no significant differences in the $O C N$ gene expression were observed among the four different titanium surfaces. We speculate that this negative result may be due to the limited time of culture in our study, as $O C N$ is only expressed by mature osteoblasts and serves as an advanced marker of osteogenic differentiation. These results demonstrate that the MNTs may be more biologically friendly, providing a more balanced promotion of multiple osteoblast functions.

Increasing evidence has demonstrated the pivotal roles of Hedgehog signaling in osteogenesis. ${ }^{6-8,12-14}$ In this regard, recent studies of biological materials have focused on the potential role of the Hedgehog pathway in mediating the response of osteoblasts to biomaterial topographies. Concerning titanium implants, several studies have shown that Hedgehog-Gli1 signaling is sensitive to the different topographical surfaces of titanium. ${ }^{18,19}$ Other studies have indicated that MNTs induce an obvious activation of several functional osteogenesis-related signaling pathways, including 
Hedgehog signaling, resulting in an enhanced osteoblast differentiation, maturation, and bone formation. ${ }^{23-25,38}$ Consequently, we conducted this study to systematically estimate the Hedgehog-Gli1 signaling response to different titanium topographies. Our data showed that the MNTs induced higher gene and protein expression of Shh, Smo, and Glil and the activation of Hedgehog signaling compared to smooth and microstructured surfaces. We speculated that the formation of numerous lamellipodia, filopodia, and cellular extensions induced by MNTs may enhance the transmembrane transmission of Hedgehog-Gli1 signaling or provide cells with an extracellular stimulus that can pass through the cytoskeletal components to the nucleoskeleton, ${ }^{39,40}$ which ultimately results in alterations in the gene expression of Hedgehog-Gli1 signaling. Further studies are required to confirm these results and investigate the specific underlying mechanisms.

The surface topographies of biomedical implants not only influence cellular functions directly via the biomaterial/ cell interaction but also affect cellular behavior indirectly by modulating mechanisms involving various functional osteogenesis-related signaling pathways. ${ }^{35,41,42}$ Our initial results indicated that MNTs upregulated the activity of the Hedgehog-Gli1 pathway through an extracellular stimulus in the microenvironment around the cell, which may ultimately result in changes to downstream events and cell functions. Several studies have demonstrated the crucial roles of the Hedgehog-Gli1 pathway in various development processes, such as osteoblast proliferation and differentiation, which are essential for bone formation. ${ }^{6,43-45}$ Oliveira et $\mathrm{al}^{46}$ discovered that the activation of the Hedgehog pathway triggered by purmorphamine in mesenchymal stem cells, as indicated by Gli1 upregulation, increased the expression of various genes (eg, Runx2 and BMPs) that are involved in osteoblast differentiation. Other genetic and cell biological studies have demonstrated that the activation of the Hedgehog pathway stimulated ALP activity and the expression of $O C N$ and Runx2. ${ }^{47-49}$ For verification, we further suppressed Hedgehog-Gli1 signaling using the Smo inhibitor cyclopamine and, then, monitored the proliferative activity, ALP production, and osteogenesis-related gene expression to determine the role of the Hedgehog-Gli1 pathway in promoting the effects of MNTs on MG63 proliferation and differentiation. Our results showed that cyclopamine significantly suppressed the gene and protein expression of Smo and Gli1 nuclear accumulation, which was consistent with previous studies. ${ }^{50,51}$ Treatment with cyclopamine decreased the proliferative activity of MG63 cells, especially the cells seeded onto MNTs. Furthermore, the enhanced expressions of the osteogenesis-related genes (BMP-2, ALP, and Runx2) and ALP production enabled by the MNTs were significantly downregulated by the presence of cyclopamine to a similar level as those on the smooth and acid-etched microstructured surfaces in the absence of cyclopamine. However, no significant differences in the $O C N$ expression levels were observed between the cyclopamine-treated and untreated cells seeded onto the four different titanium surfaces. These data confirm our hypothesis that Hedgehog-Gli1 signaling mediates the enhanced proliferation and differentiation of MG63 osteoblasts enabled by MNTs.

\section{Conclusion}

Using a hierarchical MNT model produced by common acid etching followed by anodization with $\mathrm{TiO}_{2}$ nanotubes, the present study investigated the potential role of the HedgehogGli1 signaling pathway in the response of MG63 osteoblasts to MNTs. Our results showed that the MNTs resulted in better osseointegration in vitro; this enhanced effect of MNTs on osteoblast proliferation and differentiation may be mediated by Hedgehog-Gli1 signaling. Our results greatly advance our understanding of the mechanism involved in the biological responsiveness of biomaterial topographies, which may provide insight for the optimization of hierarchical biomaterial topographies targeting Hedgehog-Gli1 signaling.

\section{Acknowledgment}

This study was supported by funding from the Science and Technology Plan Projects funds of Taishan City (20151221).

\section{Disclosure}

The authors report no conflicts of interest in this work.

\section{References}

1. Gao X, Zhang X, Song J, et al. Osteoinductive peptide-functionalized nanofibers with highly ordered structure as biomimetic scaffolds for bone tissue engineering. Int J Nanomedicine. 2015;10:7109-7128.

2. Reznikov N, Shahar R, Weiner S. Three-dimensional structure of human lamellar bone: the presence of two different materials and new insights into the hierarchical organization. Bone. 2014;59:93-104.

3. Li Y, Qi Y, Gao Q, et al. Effects of a micro/nano rough strontium-loaded surface on osseointegration. Int J Nanomedicine. 2015;10:4549-4563.

4. Wang W, Zhou S, Guo L, Zhi W, Li X, Weng J. Investigation of endocytosis and cytotoxicity of poly-d,l-lactide-poly(ethylene glycol) micro/nano-particles in osteoblast cells. Int J Nanomedicine. 2010;5: 557-566.

5. Zhao L, Liu L, Wu Z, Zhang Y, Chu PK. Effects of micropitted/ nanotubular titania topographies on bone mesenchymal stem cell osteogenic differentiation. Biomaterials. 2012;33(9):2629-2641.

6. Jemtland R, Divieti P, Lee K, Segre GV. Hedgehog promotes primary osteoblast differentiation and increases PTHrP mRNA expression and iPTHrP secretion. Bone. 2003;32(6):611-620. 
7. Wicking C, Smyth I, Bale A. The Hedgehog signalling pathway in tumorigenesis and development. Oncogene. 1999;18(55):7844-7851.

8. Yang J, Andre P, Ye L, Yang YZ. The Hedgehog signalling pathway in bone formation. Int J Oral Sci. 2015;7(2):73-79.

9. Lees C, Howie S, Sartor RB, Satsangi J. The Hedgehog signalling pathway in the gastrointestinal tract: implications for development, homeostasis, and disease. Gastroenterology. 2005;129(5): 1696-1710.

10. Agren M, Kogerman P, Kleman MI, Wessling M, Toftgard R. Expression of the PTCH1 tumor suppressor gene is regulated by alternative promoters and a single functional Gli-binding site. Gene. 2004;330 $101-114$.

11. Mas C, Ruiz i Altaba A. Small molecule modulation of HH-GLI signaling: current leads, trials and tribulations. Biochem Pharmacol. 2010;80(5):712-723.

12. Long F, Chung UI, Ohba S, McMahon J, Kronenberg HM, McMahon AP. Ihh signaling is directly required for the osteoblast lineage in the endochondral skeleton. Development. 2004;131(6):1309-1318.

13. Wang Q, Huang C, Zeng F, Xue M, Zhang X. Activation of the Hh pathway in periosteum-derived mesenchymal stem cells induces bone formation in vivo: implication for postnatal bone repair. Am J Pathol. 2010;177(6):3100-3111.

14. Kitaura Y, Hojo H, Komiyama Y, Takato T, Chung UI, Ohba S. Gli1 haploinsufficiency leads to decreased bone mass with an uncoupling of bone metabolism in adult mice. PLoS One. 2014;9(10):e109597.

15. Grande DA, Mason J, Light E, Dines D. Stem cells as platforms for delivery of genes to enhance cartilage repair. J Bone Joint Surg Am. 2003; 85-A(suppl 2):111-116.

16. Kawai S, Sugiura T. Characterization of human bone morphogenetic protein (BMP)-4 and -7 gene promoters: activation of BMP promoters by Gli, a sonic hedgehog mediator. Bone. 2001;29(1):54-61.

17. Roelink H. Tripartite signaling of pattern: interactions between Hedgehogs, BMPs and Wnts in the control of vertebrate development. Curr Opin Neurobiol. 1996;6(1):33-40.

18. Donos N, Retzepi M, Wall I, Hamlet S, Ivanovski S. In vivo gene expression profile of guided bone regeneration associated with a microrough titanium surface. Clin Oral Implants Res. 2011;22(4):390-398.

19. Kim SY, Yoo JY, Ohe JY, et al. Differential expression of osteomodulatory molecules in periodontal ligament stem cells in response to modified titanium surfaces. Biomed Res Int. 2014;2014:452175.

20. Lin GL, Hankenson KD. Integration of BMP, Wnt, and notch signaling pathways in osteoblast differentiation. J Cell Biochem. 2011;112(12): 3491-3501.

21. Regard JB, Malhotra D, Gvozdenovic-Jeremic J, et al. Activation of Hedgehog signaling by loss of GNAS causes heterotopic ossification. Nat Med. 2013;19(11):1505-1512.

22. Tavella S, Biticchi R, Schito A, et al. Targeted expression of SHH affects chondrocyte differentiation, growth plate organization, and Sox9 expression. J Bone Miner Res. 2004;19(10):1678-1688.

23. Miron RJ, Shuang Y, Bosshardt DD, Caballe-Serrano J, Chandad F, Zhang Y. Osteogenic gene array of osteoblasts cultured on a novel osteoinductive biphasic calcium phosphate bone grafting material. Clin Oral Investig. 2017;21(3):801-808.

24. Kaur G, Valarmathi MT, Potts JD, Jabbari E, Sabo-Attwood T, Wang Q Regulation of osteogenic differentiation of rat bone marrow stromal cells on 2D nanorod substrates. Biomaterials. 2010;31(7):1732-1741.

25. Kaur G, Valarmathi MT, Potts JD, Wang Q. The promotion of osteoblastic differentiation of rat bone marrow stromal cells by a polyvalent plant mosaic virus. Biomaterials. 2008;29(30):4074-4081.

26. Wang W, Liu Q, Zhang Y, Zhao L. Involvement of ILK/ERK1/2 and ILK/p38 pathways in mediating the enhanced osteoblast differentiation by micro/nanotopography. Acta Biomater. 2014;10(8):3705-3715.

27. Wang $\mathrm{W}, \mathrm{Zhao} \mathrm{L}, \mathrm{Wu} \mathrm{K}$, et al. The role of integrin-linked kinase/ beta-catenin pathway in the enhanced MG63 differentiation by micro/ nano-textured topography. Biomaterials. 2013;34(3):631-640.

28. Olivares-Navarrete R, Hyzy SL, Hutton DL, et al. Role of non-canonical Wnt signaling in osteoblast maturation on microstructured titanium surfaces. Acta Biomater. 2011;7(6):2740-2750.
29. Mohseny AB, Cai Y, Kuijjer M, et al. The activities of Smad and Gli mediated signalling pathways in high-grade conventional osteosarcoma. Eur J Cancer. 2012;48(18):3429-3438.

30. Thayer SP, di Magliano MP, Heiser PW, et al. Hedgehog is an early and late mediator of pancreatic cancer tumorigenesis. Nature. 2003;425(6960): 851-856.

31. Wu X, Wang S. Regulating MC3T3-E1 cells on deformable poly(epsiloncaprolactone) honeycomb films prepared using a surfactant-free breath figure method in a water-miscible solvent. ACS Appl Mater Interfaces. 2012;4(9):4966-4975.

32. Wu X, Wang S. Biomimetic calcium carbonate concentric microgrooves with tunable widths for promoting MC3T3-E1 cell functions. $A d v$ Healthc Mater. 2013;2(2):326-333.

33. Boyan BD, Lossdorfer S, Wang L, et al. Osteoblasts generate an osteogenic microenvironment when grown on surfaces with rough microtopographies. Eur Cell Mater. 2003;6:22-27.

34. Kim MJ, Kim CW, Lim YJ, Heo SJ. Microrough titanium surface affects biologic response in MG63 osteoblast-like cells. J Biomed Mater Res A. 2006;79(4):1023-1032.

35. Schwartz Z, Olivares-Navarrete R, Wieland M, Cochran DL, Boyan BD. Mechanisms regulating increased production of osteoprotegerin by osteoblasts cultured on microstructured titanium surfaces. Biomaterials. 2009;30(20):3390-3396.

36. Ding X, Zhou L, Wang J, et al. The effects of hierarchical micro/ nanosurfaces decorated with $\mathrm{TiO}_{2}$ nanotubes on the bioactivity of titanium implants in vitro and in vivo. Int $J$ Nanomedicine. 2015;10: 6955-6973.

37. Liang J, Xu S, Shen M, et al. Osteogenic activity of titanium surfaces with hierarchical micro-/nano-structures obtained by hydrofluoric acid treatment. Int J Nanomedicine. 2017;12:1317-1328.

38. Nikukar H, Reid S, Tsimbouri PM, Riehle MO, Curtis AS, Dalby MJ. Osteogenesis of mesenchymal stem cells by nanoscale mechanotransduction. ACS Nano. 2013;7(3):2758-2767.

39. Dalby MJ, Biggs MJ, Gadegaard N, Kalna G, Wilkinson CD, Curtis AS Nanotopographical stimulation of mechanotransduction and changes in interphase centromere positioning. $J$ Cell Biochem. 2007;100(2): 326-338.

40. Dalby MJ, Gadegaard N, Herzyk P, et al. Nanomechanotransduction and interphase nuclear organization influence on genomic control J Cell Biochem. 2007;102(5):1234-1244.

41. Gu YX, Du J, Si MS, Mo JJ, Qiao SC, Lai HC. The roles of PI3K/Akt signaling pathway in regulating MC3 3 3-E1 preosteoblast proliferation and differentiation on SLA and SLActive titanium surfaces. J Biomed Mater Res A. 2013;101(3):748-754.

42. Wang W, Zhao L, Ma Q, Wang Q, Chu PK, Zhang Y. The role of the Wnt/beta-catenin pathway in the effect of implant topography on MG63 differentiation. Biomaterials. 2012;33(32):7993-8002.

43. Felber K, Croucher P, Roehl HH. Hedgehog signalling is required for perichondral osteoblast differentiation in zebrafish. Mech Dev. 2011; 128(1-2):141-152.

44. Huycke TR, Eames BF, Kimmel CB. Hedgehog-dependent proliferation drives modular growth during morphogenesis of a dermal bone. Development. 2012;139(13):2371-2380.

45. St-Jacques B, Hammerschmidt M, McMahon AP. Indian hedgehog signaling regulates proliferation and differentiation of chondrocytes and is essential for bone formation. Genes Dev. 1999;13(16): 2072-2086.

46. Oliveira FS, Bellesini LS, Defino HL, da Silva Herrero CF, Beloti MM, Rosa AL. Hedgehog signaling and osteoblast gene expression are regulated by purmorphamine in human mesenchymal stem cells. J Cell Biochem. 2012;113(1):204-208.

47. Shimoyama A, Wada M, Ikeda F, et al. Ihh/Gli2 signaling promotes osteoblast differentiation by regulating Runx2 expression and function. Mol Biol Cell. 2007;18(7):2411-2418.

48. Wu X, Walker J, Zhang J, Ding S, Schultz PG. Purmorphamine induces osteogenesis by activation of the hedgehog signaling pathway. Chem Biol. 2004;11(9):1229-1238. 
49. Yuasa $\mathrm{T}$, Kataoka H, Kinto N, et al. Sonic hedgehog is involved in osteoblast differentiation by cooperating with BMP-2. J Cell Physiol. 2002;193(2):225-232.

50. Warzecha J, Gottig S, Chow KU, et al. Inhibition of osteosarcoma cell proliferation by the Hedgehog-inhibitor cyclopamine. JChemother. 2007; 19(5):554-561.
51. Zhu SL, Luo MQ, Peng WX, et al. Sonic hedgehog signalling pathway regulates apoptosis through Smo protein in human umbilical vein endothelial cells. Rheumatology (Oxford). 2015;54(6):1093-1102.

\section{Publish your work in this journal}

The International Journal of Nanomedicine is an international, peerreviewed journal focusing on the application of nanotechnology in diagnostics, therapeutics, and drug delivery systems throughout the biomedical field. This journal is indexed on PubMed Central, MedLine, CAS, SciSearch $®$, Current Contents ${ }^{\circledR} /$ Clinical Medicine,
Journal Citation Reports/Science Edition, EMBase, Scopus and the Elsevier Bibliographic databases. The manuscript management system is completely online and includes a very quick and fair peer-review system, which is all easy to use. Visit http://www.dovepress.com/ testimonials.php to read real quotes from published authors. 